\title{
Applications Enabled by a Deterministic Wireless Communication System Such as Industrial LTE
}

\author{
Philipp Neher ${ }^{1+}$, Armin Lechler ${ }^{1}$ and Alexander Verl ${ }^{1}$ \\ ${ }^{1}$ Institute for Control Engineering of Machine Tool and Manufacturing Units, University of Stuttgart, \\ Stuttgart, Germany
}

\begin{abstract}
Current industrial communication systems rely heavily on communication cables to transmit information and control signals. Wireless networks are emerging on the factory level with non-real-time requirements. The needed cable for deterministic real-time communication hinders the development and deployment of various applications in the production environment. Some of these applications might be an integral part in solving the challenges of future production. Along with a description of a wireless communication system, various examples for enabled applications are presented in this paper.
\end{abstract}

Keywords: wireless communication, automated guided vehicles, industrial LTE

\section{Introduction}

One goal of the Internet of Things (IoT) is the interconnection of everything. The promise of networking different machines and sensors together and having them exchange and share various information represents an overall improved efficiency and in some cases improved effectivity [1]. This way, there is a better use of resources (e.g. energy, time) and the resources might last longer. Also new sources of natural resources can be exploited. In addition, networked devices make it possible to introduce automation to various tedious tasks of our daily living. Therefore the level of comfort of our life is raised.

Apart from the private domain, the IoT arrived in the producing industry. The producing industry already utilizes a lot of automation but hopes to further improve productivity or efficiency through the concepts of the IoT. Thus more distributed sensors are introduced and interconnected. Additionally smaller components of industrial machines are equipped with more intelligence and computational power. These intelligent components form together larger units, so called "Cyber-Physical Systems (CPS)". CPS can be stationary or mobile and will be an integral element of the smart factories of the future [2]. With mobile CPS smart factories are able to adapt themselves and change the production layout following fluctuating demand and a range of products [3].

While stationary CPS and sensors can be interconnected using cable based networks, cables are usually not suited for highly mobile applications. Therefore CPS must be networked wirelessly [2]. In the private domain, IoT enabled devices utilize a range of different wireless communication systems. There IoT is used for automating tasks but, the offered applications have different requirements compared to CPS and industrial automation. Currently no wireless communication system addresses all of the industrial requirements of CPS to satisfaction [4]. Still, a real time capable industrial wireless communication system would enable a multitude of applications not possible today.

The paper is outlined as follows: The main requirements of industrial wireless communication systems in comparison to exiting wireless systems are discussed in the following section. In section III a proposed

\footnotetext{
+ Corresponding author. Tel.: +49 711685 84499; fax: +4971168574499.

E-mail address: philipp.neher@isw.uni-stuttgart.de.
} 
wireless system is described. Some applications made possible through such a wireless communication systems are presented in Section IV. Section V concludes the paper with a summary and future outlook.

\section{Requirements of Industrial Communication}

Mobile CPS will be located in or form together productions cells. Therefore the requirements concerning communication will be the same for mobile CPS and productions cells. In [4] the communication requirements for productions cells are given. Within a production cell a deterministic data transmission in hard or isochronous real time is required. For determinism a reliable communication is necessary [5]. The Bit Error Rate (BER) must be very low for a reliable communication without the need to request retransmission. Retransmission requests are unfavourable because they increase latency and thus destroy time determinism if latency varies too much. For cooperated movement of axes a synchronized communication scheme is required [6]. Set point data must be at the axis controllers in time for a synchronous execution of movement. Also industrial control systems expect a constant bandwidth of their communication systems.

The authors compare these requirements with the properties of available wireless communication systems in [7]. It is concluded that none of the investigated systems meets all the necessary values. While most wireless communication systems offer a data bandwidth and a range that is large enough for industrials applications they fail in terms of reliability and stability. Further not all systems do offer the necessary reaction or cycle time, and none can keep a constant cycle time short enough for industrial control.

\section{Proposed Wireless Communication System}

In [8] the authors offer a concept for an industrial wireless communication system based on LTE Advanced. The cellular communication standard LTE Advanced [9] can deliver a large amount of data to its users. It also offers bandwidth adaption to cope with the varying quality of the wireless link. This increases stability because users are not dropped as soon as interferences and disturbances deteriorate the wireless link. Additionally the minimal possible cycle time of LTE Advanced is within the required range for industrial control applications. For improvement of the BER the usage of an application layer-based forward error correction (AL-FEC) [10] scheme is proposed. This way the basic LTE Advanced system is not changed and standard hardware might be deployed. Utilization of a GPS-based clock synchronization method [11] is proposed to solve the problem of synchrony of the communication. By having a synchronized clock in every wireless connected device, the execution of actions can be based on this common timestamp instead of the varying arrival of a message. This way only the maximum latency of the wireless transmission must be known (or defined) and adhered. To accommodate the adaptive bandwidth the concept includes a streaming mechanism. This could be modelled after the dynamic adaptive streaming over HTTP (DASH) mechanism [12] which is used for delivering video to mobile devices. The control data can be categorized and divided into various levels of importance and timeliness factors [13]. Then the transmission of the categorized data adapted to the available bandwidth, sending always important data and only sending less important data when bandwidth is available. Lastly all the different parts and mechanisms should be implemented on a FPGA and mostly in hardware to keep the overall latency as low as possible. The implemented variant of the respective schemes (AL-FEC, GPS-Sync., Streaming) need to be chosen accordingly for the same reason.

\section{Enables Applications}

A time deterministic wireless communication system as the one described in the previous section could be a wireless fieldbus in analogy to the wired communications systems presented in today's producing industry. They pave the way for further applications that currently are not possible. In the following, some enabled applications and uses are presented. Obviously the examples include applications based on future mobile CPS, but uses in current automated guided vehicle systems (AGVS) are also outlined. Additional examples of other applications are introduced as well.

\subsection{Cyber Physical Systems}

With the term "CPS" not clearly defined, it is hard to tell what CPS really, are and if CPS are already present in the production systems. Therefore the detailed capabilities and tasks of CPS are currently 
unknown. Such being the case, this section understands mobile CPS are intelligent mobile working platforms which act at least partly autonomous.

General purpose mobile industrial robots: An industrial robot is a universal tool that can handle a lot of different tasks. But the size of its workspace is limited. If an industrial robot is outfitted with wheels or some other form of movement system and is controlled and powered wirelessly, the size of its workspace becomes almost unlimited, and it becomes even more universal. These kind of devices are already under development [14].

Cooperative production between mobile CPS and stationary machine: Mobile CPS will have to work together with stationary machines. These can be more simple tasks like loading tooling machines, but can also mean cooperative processing of a workpiece. For example, when a workpiece is held by the CPS and a tool is operated by a stationary machine, a synchronous movement is imminent.

Cooperative tasks between multiple CPS: The same as the example above, CPS are not limited to collaborate with stationary machines only. A wireless fieldbus would allow two or more CPS to cooperate with each other.

Shared Workspaces: To avoid collisions between multiple CPS, one approach is to have them stay outside of each other's workspaces. This is not always an efficient usage of the available space. When sharing workspaces the CPS need to be aware of each other's positions, motions and actions. This could be realized by sensors on the CPS [15]. Another way would be to have each CPS communicate all that information of their own state and action to the other CPS sharing their workspace.

\subsection{Automated Guided Vehicle Systems (AGVS)}

While CPS are not a common sight in production environments yet, automated guided vehicle systems are used widely to transport workpieces and material in factories already. Most systems rely on some kind of guidance mechanism like visible or inductive lines on the floor. The flexibility of these systems is therefore limited because the guidance systems are usually static. With a deterministic wireless communication system, flexible guidance systems become feasible allowing for a free motion of AGVS.

External pathfinding and control: Usually an AGVS is used to transport material from point A to point B. When the path between those points is not predetermined by a fixed guidance system, a path needs to be found by the AGVS using for example a map. In static factory layouts this could be done on-board the AGVS, but requires processing power which might be too much for the on-board processing system. Therefore the pathfinding could be done externally on a stationary central processing system and the computed path then communicated to the AGVS. Furthermore, the drive control could even be offloaded to a centralized control and the AGVS then driven remote controlled. The central control could reside inside a cloud-based system and control multiple AGVS to potentially save installed resources. The feasibility of such an approach has been shown in [16].

External sensors: If autonomous vehicles are deployed in a changing environment, static maps and absolute position tracking are not sufficient. The vehicle needs additional sensors to determine its current surroundings. In factories theses sensors do not need to be on-board the AGVS if a wireless fieldbus is available. External sensors could be installed in the factory infrastructure and the data then communicated to the AGVS.

External sensor computation: Sensors used to perceive the vicinity of an AGVS produce lots of raw data. This raw data must be filtered and processed to be used for pathfinding and collision detection methods. If the on-board processing unit is capable enough this can be done on-board the AGVS. In other cases the computation needs to be offloaded to another (probably stationary and centralized) processing system. The results are subsequently communicated back to the AGVS.

Environment mapping and map sharing: Freely moving AGVS need to map the environment they are moving in to be able to find their way without colliding with obstacles. The detection range of the sensors is limited, so the size or the actuality of the maps is also limited if the AGVS is operating individually. Multiple AGVS could share their maps wirelessly to chart larger areas and keep the maps more up to date. 
Cooperative transport: For the transportation of a large or heavy workpiece a large AGVS is currently needed. If the movement of multiple AGVS can be executed synchronously, multiple smaller AGVS could transport large or heavy workpieces while cooperating with each other. This way a centralized control coordinates multiple AGVS to perform a task a single AGVS could not handle.

Swarm intelligence: Multiple wirelessly interconnected AGVS could distribute given tasks among themselves without the need for a centralized control. They could adjust the path on which they plan to move to the path of the other AGVS to avoid collisions far in advance. In this case the multiple AGVS decide between each other which single AGVS handles which task. No centralized control instructs single AGVS to do a single task but gives all the tasks (which a single AGVS could handle) to the whole swarm.

Factory-wide control: Deterministic wireless communication systems are required to transmit important information to all AGVS in a factory or parts of a factory. For example, the maximum allowed velocity of all AGVS could be reduced if a person is entering specific areas, or in the case of an incident. Also the integration of the AGVS into a central safety system would be possible with the ability to communicate an emergency stop command to the AGVS.

\subsection{Warehouse, and Storage and Retrieval Vehicles}

Large vertical warehouses are operated by autonomous storage and retrieval vehicles (ASRV) which are similar to AGVS. With deterministic wireless communication systems new possibilities for ASRV unfold.

Multiple ASRV: Without the need of a static cable connection and the option to directly communication between multiple ASRV it is feasible to deploy multiple ASRV on the same track. This way they could then cooperate with each other to transport large or heavy materials. Further, the whole system would be more flexible and have an increased performance when the ASRV operate separately, but can avoid collisions because they communicate each other's positions and actions.

Modular intelligent warehouses: When increasing the production quantity, usually the warehouse storage capability also needs to be increased. This could be realized by intelligent storage modules. Each storage module has in internal storage system to store or retrieve stock. An external ASRV acts as interface between the storage modules. Through deterministic wireless communication the internal storage system can communicate with the external ASRV and a centralized control.

\subsection{Modular Reconfigurable Factories}

Because of increasing fluctuation of demand, and individualization of products and therefore larger variant numbers, future factories need to be more adaptable. Deterministic wireless communication systems can help to increase efficiency when reconfiguring a factory or shop floor. Without the requirement of a physical connection for communication the needed cost and time of installing, moving and setting up a machine is reduced. Also production modules can be adaptively added or removed to the current production process to react to change of demanded production volume. With the reduced required setup time, production lines can be adapted more easily and efficiently to product changes. This would be all possible with wireless fieldbuses because no communication cables need to be laid and plugged in.

\subsection{Cable Robots}

Cable robots use multiple cables to position an effector (tool or workpiece) [17]. Often it is necessary to transmit data from or to the effector. This could be sensor readings or set points. In this application, communication cables might physically interfere with the motion cables. Also the wear on the communication cables is especially high because of the agility and velocity of cable robots.

\subsection{Mobile Human Machine Interfaces}

Human machine interfaces (HMI) can be made mobile with wireless communication. With this, an operator could control a stationary machine, CPS or AGVS from anywhere within the factory. This makes it possible for the operator to change his viewing angle on the workpiece or to follow an AGVS. The HMI could include an emergency stop if the wireless communication is deterministic and stable. This way every person on the shop floor could carry a personal emergency stop switch. Thus the need to locate and reach an installed emergency stop switch would be eliminated, resulting in a faster save state. 


\section{Conclusion and Outlook}

A deterministic wireless communication system or wireless fieldbus is an enabler technology for multiple applications, especially for Cyber Physical Systems and automated guided vehicle systems. Various examples exploiting the absence of communication cables have been presented. Additionally, an approach for a deterministic wireless communication system was described. The given examples underline the need to develop such a system. The objective of future work is to further evaluate the proposed approach through simulation and a subsequently prototypical setup.

\section{Acknowledgements}

The work presented in this paper was funded by the German Research Foundation (DFG).

\section{References}

[1] A. P. Athreya and P. Tague. Network self-organization in the Internet of Things. In: 2013 IEEE International Conference on Sensing, Communications and Networking (SECON), New Orleans, LA, 2013, pp. 25-33.

[2] Acatech. Cyber-Physical Systems. Innovationsmotoren für Mobilität, Gesundheit, Energie und Produktion (acatech POSITION). Springer Verlag, Heidelberg, 2011.

[3] H. Kagermann, W. Wahlster and J. Helbig. Recommendations for implementing the strategic initiative INDUSTRIE 4.0 - Final report of the Industrie 4.0 Working Group. Acatech, 2013.

[4] VDI/VDE 2185. Radio based communication in industrial automation. VDI/VDE 2185, Beuth Verlag, Berlin, 2007.

[5] H. Kopetz, A. Ademaj, P. Grillinger and K. Steinhammer. The Time-triggered Ethernet (TTE) Design. In: Proceedings of the IEEE International Symposium on Object-Oriented Real-Time Distributed Computing. 2005.

[6] R.S.H. Piggin. Developments in Real-time Control with Ethernet. In: 6th International Technology and Innovation Conference. ITC, 2006.

[7] P. Neher, A. Lechler and A. Verl. Industrielles LTE. In: Fortschrittsberichte VDI, Reihe 2, Fertigungstechnik. VDI-Verlag, Düsseldorf, in press.

[8] P. Neher and A. Lechler. Concept for Real-Time Wireless Networking of Mobile CPS Based on LTE. In: Advanced Materials research. Trans Tech Publications, Switzerland, Vol. 1140, 2016.

[9] K. Loa, et al.. IMT-Advanced Relay Standards. IEEE Communications Magazine, 2010.

[10] T. Stockhammer, A. Shokrollahi, M. Watson, M. Luby and T. Gasiba. Application Layer Forward Error Correction for Mobile Multimedia Broadcasting. In: Handbook of Mobile Broadcasting: DVB-H. DMB, ISDB-T and Media FLO, p. 239-280, CRC Press, 2008.

[11] D. Mazur, R. D. Quint and V. A. Centeno. Time Synchronization of Automation Controllers for Power Applications. In: IEEE Transactions on Industry Application. Vol. 50, No. 1, 2014.

[12] N.N.. ISO/IEC 23009-1: 2012 Information Technology-Dynamic adaptive streaming over HTTP (DASH)-Part 1: Media presentation description and segment formats. 2012.

[13] J. Schlechtendahl, F. Kretschmer, A. Lechler and A. Verl., Communication mechanisms for cloud based machine controls. In: 47th CIRP Conference on manufacturing Systems. Windsor, Canada, 2014.

[14] J. Saenz, et al.. Valeri - a collaborative mobile manipulator for aerospace production. In: Advances in Cooperative Robotics, Proceedings of the 19th International Conference on CLAWAR. 2016.

[15] A. Csiszar, M. Drust, T.Dietz, A. Verl and C. Brisan. Dynamic and Interactive Path Planning and Collision Avoidance for an Industrial Robot Using Artificial Potential field Based Method. In: Mechatronics: Recent Technological and Scientific Advances, Part IV. Springer, Berlin, 2012.

[16] F. Kretschmer, S. Friedl, A. Lechler and A. Verl. Communication extension for cloud-based machine control of simulated robot processes. In: 2016 IEEE International Conference on Industrial Technology (ICIT). Taipei, 2016.

[17] P. Tempel, P. Miermeister and A. Pott. Kinematics and Dynamic Modeling for Real-Time Simulation of the Cable-Driven Parallel Robot IPAnema 3. In: 14th World Congress in Mechanism and Machine Science. Taipei, Taiwan, 2015. 